\title{
Monitorización de la perfusión tisular en el paciente criticamente enfermo
}

\section{Monitoring tissue perfusion in critically ill patients}

\author{
Hernández-González Guadalupe Lisseth ${ }^{1}$, Salgado Reyes, José Manuel²
}

\section{RESUMEN}

La hipoperfusión tisular contribuye a la disfunción orgánica múltiple, por lo que deberá de ser monitoreada en los pacientes críticamente enfermos; El choque de cualquier etiología, se caracteriza por la inadecuada perfusión de los tejidos del organismo, produciendo una situación de desequilibrio entre el aporte y la demanda de oxígeno. Sin embargo, la evaluación hemodinámica temprana que se lleva a cabo mediante hallazgos físicos, entre ellos signos vitales, presión venosa central y volumen urinario no detectan la hipoxia tisular global persistente.

La monitorización actual para la valoración de la oxigenación tisular durante la reanimación del paciente crítico está basada principalmente en los parámetros de transporte y consumo de oxígeno derivados de la hemodinámica global.

\section{ABSTRACT}

Tissue hypoperfusion contributes to multiple organ dysfunction, so it should be monitored in critically ill patients; The shock of any etiology, is characterized by the inadequate tissue perfusion, producing a situation of imbalance between the contribution and the oxygen demand. However, early hemodynamic evaluation using physical findings, including vital signs, central venous pressure and urinary volume, does not detect persistent global tissue hypoxia. The current monitoring for evaluation of tissue oxygenation during critical patient resuscitation is based mainly on oxygen transport and oxygen consumption parameters derived from global hemodynamics.

\section{INTRODUCCIÓN}

$\mathrm{L}$

a oxigenación tisular se define como el aporte de

Joxígeno adecuado a la demanda. La demanda de oxígeno depende de los requerimientos metabólicos de cada tejido ${ }^{1}$.

Los pacientes críticamente enfermos presentan un estado de hipermetabolismo secundario a la respuesta neuroendócrina al estrés, caracterizada por la secreción de cortisol, catecolaminas y citosinas que provocan glucólisis acelerada, gluconeogénesis excesiva, glucogenólisis y resistencia a la insulina, así como aumento de la degradación de proteínas y del catabolismo de las grasas. Este fenómeno que ocurre durante la fase aguda, suele dar como resultado la presencia de hiperglucemia ${ }^{2}$ típicamente asociada al aumento del consumo de oxígeno ${ }^{3}$ así como a una respuesta cardiovascular hiperdinámica. ${ }^{4}$

Cualquier paciente ante una lesión aguda, puede presentar disfunción celular secundaria a hipoperfusión e hipoxia a nivel tisular. La inestabilidad hemo- dinámica aunada a hipoxia citopática por disfunción mitocondrial en dichos pacientes, pueden desencadenar disfunción organica múltiple de manera progresiva hasta llevar a la muerte. ${ }^{5}$

La monitorización hemodinámica durante la fase aguda del shock será útil en la determinación y el tratamiento de las alteraciones fisiopatológicas en las enfermedades críticas así como en la evaluación de la respuesta del paciente a la reanimación, por lo que las variables utilizadas deberán valorar la persistencia o resolución de la hipoxia tisular.6

En la actualidad, las variables más estudiadas en cuanto a detección de hipoxia tisular son el lactato en sangre y las saturaciones venosas de oxígeno ( $\mathrm{SvO} 2$ y SvcO2). ${ }^{7}$ Otros autores han encontrado que el delta de $\mathrm{CO} 2$ tiene buena sensibilidad como marcador de perfusión y que este tiene asociación con la mortalidad en los pacientes en estado de choque. ${ }^{8,9}$
'Estudiante de Medicina, Universidad Autónoma del Estado de México

${ }^{2}$ Médico especialista en $\mathrm{Me}$ dicina Crítica. Centro Médico ISSEMYM

Correspondencia a: Hernández González Guadalupe Lisseth

Correo electrónico: lisseth.hernandezg@gmail.com

Palabras clave: Perfusión tisular, choque, monitoreo hemodinámico, reanimación.

Keywords: Tissue perfusion, shock, hemodynamic monitoring, resuscitation.

Procedencia y arbitraje: no comisionado, sometido a arbitraje externo.

Recibido para publicación: 02 de Septiembre de 2016 Aceptado para publicación: 09 de Dlciembre de 2016

Citar como: Rev Cient Cienc Med 2016; 19(2): 43-47 


\section{METOdología de BÚSQUEDA DE ARTÍCULOS}

Se realizó la búsqueda en las bases de datos Pubmed, Critical Care, Medline, Embase y Scopus, usando las palabras clave "tissue perfusion", "shock", "perfusion markers", "lactate","pressure perfusion" y "hemodynamic resuscitation". Se priorizaron las publicaciones de los últimos cinco años, sin embargo se incluyeron artículos desde el 01 de enero del 2000 hasta el 31 de octubre de 2016 en la lista final de referencias. Sólo se analizaron ensayos clínicos aleatorios, estudios comparativos, estudios observacionales y de revisión bibliográfica; Se excluyeron los comentarios y los editoriales, así como los estudios en animales y los estudios publicados en otros idiomas distintos del inglés y el español.

\section{DESARROLLO}

En la práctica de la medicina crítica es necesario asegurar el bienestar de los órganos y tejidos mientras se trata la enfermedad de base del paciente. Para que dicha acción se lleve a cabo, es necesario asegurar el transporte de oxígeno y nutrientes a la célula para que esta realice sus funciones básicas.

Cualquier lesión aguda puede condicionar hipoxemia a nivel tisular y con ello disfunción celular. En la hipoxemia grave, al existir una disminución del aporte de oxígeno, el consumo de oxígeno se mantiene gracias a la compensación determinada por el aumento en el porcentaje de extracción. ${ }^{10} \mathrm{El}$ aporte crítico de oxígeno calculado es de $4 \mathrm{ml} / \mathrm{kg} / \mathrm{min} .{ }^{11}$ A nivel local, ocurren una serie de procesos de autorregulación que conducen a la liberación de sustancias vasodilatadoras como la prostaciclina, el óxido nítrico o el factor hiperpolarizante dependiente del endotelio, en respuesta al descenso de la presión de oxígeno. ${ }^{3}$

Cuando el porcentaje de extracción de oxígeno excede su punto crítico, ocurre un aumento del metabolismo anaerobio con lo que se elevan los niveles séricos de lactato, hidrogeniones y fosfatos inorgánicos en la célula, causando acidosis láctica. ${ }^{12}$ Debido a estos cambios hemodinámicos en el paciente gravemente enfermo, es posible utilizar marcadores de perfusión tisular. A continuación se describen los parámetros más utilizados para la detección de hipoxia tisular y su uso como objetivos en la reanimación hemodinámica.

Parámetros hemodinámicos

1.Presión arterial media (PAM)

El intercambio de gases a nivel tisular no depende solo del contenido de oxígeno arterial sino de una adecuada presión de perfusión en el tejido. La presión arterial media está determinada por la resistencia periférica total y el gasto cardíaco. Cualquier alteración a estos niveles generará hipoperfusión y ésta podrá tener como resultado insuficiencia orgánica múltiple. ${ }^{13}$

A niveles de PAM por debajo de $65 \mathrm{mmHg}$ los lechos vasculares pierden su capacidad de autorregulación local, por lo que ocurren mecanismos compensatorios; Si bien una PAM de $65 \mathrm{mmHg}$ no asegura una perfusión tisular aceptable, una PAM inferior a $65 \mathrm{mmHg}$ casi siempre traerá como consecuencia insuficiencia hemodinámica. ${ }^{14}$,

La campaña Surviving Sepsis recomienda una presión arterial media superior a $65 \mathrm{mmHg}$ en los pacientes con shock séptico. ${ }^{15}$ Los resultados del estudio SEPSISPAM ${ }^{16}$ (Sepsis y Presión Arterial Media) sugieren que un objetivo de PAM de 65 a $75 \mathrm{~mm}$ $\mathrm{Hg}$ suele ser suficiente en pacientes con choque, pero una PAM mayor (alrededor de 75 a $85 \mathrm{~mm} \mathrm{Hg}$ ) es recomendable en pacientes con hipertensión arterial crónica. Esta cuestión es de gran importancia clínica en vista de la alta prevalencia de hipertensión crónica en pacientes ingresados en unidades de cuidados intensivos.

2.Saturaciones venosas de oxígeno (Central y mixta)

La saturación venosa central de oxígeno $(\mathrm{SvcO} 2)$ y la saturación venosa mixta de oxígeno $(\mathrm{SvO} 2)$ se obtienen midiendo la saturación de oxígeno en la sangre venosa que regresa al corazón. Estas variables están determinadas por el suministro de oxígeno (flujo macrocirculatorio), distribución (flujo microcirculatorio) y procesamiento (función mitocondrial) ${ }_{1}^{17}$ por lo que evalúan de manera integral la relación entre el aporte y el consumo de oxígeno. En general, los valores menores del 60\%-65\% en el enfermo agudo, deben alertar sobre la presencia de hipoxia tisular o perfusión inadecuada y deben instar a que se realicen más diagnósticos y acciones apropiadas ${ }^{18}$, mientras que los valores normales o altos no descartan la hipoxia persistente de los tejidos. ${ }^{19}$

Clinicamente, es más sencillo obtener la $\mathrm{SvO} 2$, evitando así la cateterización de la arteria pulmonar; sin embargo en pacientes críticamente enfermos, la sustitución de $\mathrm{SvO} 2$ por $\mathrm{ScvO} 2$ resulta en una gran variabilidad. ${ }^{20,21}$ Esto podría explicarse en parte por las modificaciones de la distribución del flujo sanguíneo y la extracción de oxígeno por el tejido cerebral y esplácnico. ${ }^{22}$

En conclusión, las saturaciones venosas de oxígeno valoran de manera indirecta la perfusión tisular al integrar el estado cardiopulmonar y hemodinámico 
de los enfermos graves, por lo que la mayoría de los estudios concluyen que debe formar parte de la monitorización en las salas de cuidados intensivos. ${ }^{23}$

3.Lactato

El lactato es un metabolito crucial en los principales procesos de producción de ATP, siendo un producto del metabolismo anaerobio.

$\mathrm{Al}$ presentarse una reducción del suministro sistémico de oxígeno (nivel de hemoglobina, saturación de oxígeno y gasto cardiaco) que excede la capacidad de extracción de oxígeno, se genera hipoxia tisular, lo que aumenta el metabolismo anaerobio y conduce al aumento en los niveles séricos de lactato. Debido a que el intercambio de oxígeno tiene lugar en la microcirculación, las alteraciones en la perfusión microcirculatoria también pueden resultar en una disponibilidad limitada de oxígeno. ${ }^{24}$ Particularmente en la sepsis, el trastorno microcirculatorio puede conducir a oxígeno insuficiente que se entrega a la célula, lo que también aumenta los niveles de lactato. Por ello, monitorizar el lactato es una forma de evaluar el metabolismo anaerobio. ${ }^{25}$

El metabolismo de la glucosa durante la hipoxia tisular da como resultado la producción de lactato, ATP y agua. La producción de $\mathrm{H}+$ se origina de la hidrólisis de ATP a ADP, lo que puede condicionar una acidosis láctica.

La concentración sérica normal de lactato varía en un rango de 0,3-1,3 $\mathrm{mmol} / \mathrm{L}$ y en general son menores a $2 \mathrm{mmol} / \mathrm{L}$ en condiciones fisiológicas 26 . Su eliminación es principalmente hepática en un 60\% (a través de gluconeogenésis y oxidación a $\mathrm{CO} 2$ y agua) y renal en un 5\%-30\% (a través de su conversión a piruvato). El porcentaje restante es eliminado por el corazón y el músculo esquelético. ${ }^{27}$

Aunque frecuentemente se utiliza para diagnosticar la oxigenación inadecuada de los tejidos, otros procesos no relacionados con la oxigenación tisular pueden aumentar los niveles de lactato. Varias condiciones clínicas se han asociado con deterioro del aclaramiento del lactato, entre ellas la disfunción hepática ${ }^{28}$ y la cirugía cardiaca. ${ }^{29}$

En general, se acepta que cifras mayores a 2 $\mathrm{mmol} / \mathrm{L}$ de lactato sérico pueden aumentar la mortalidad y empobrecer el pronóstico del paciente.30 Las guias "Surviving Sepsis" utilizan el lactato sérico como criterio diagnóstico de sepsis y mencionan un mayor aumento en la mortalidad cuando éste se encuentra por encima de $4 \mathrm{mmol} / \mathrm{L} .15$

4.Delta de $\mathrm{CO} 2$

Diariamente se producen cerca de 15,000 y 20,000 mmol. De CO2. El balance de CO2 se logra cuando la cantidad producida por el metabolismo celular es transportada por la circulación y excretada por los pulmones. ${ }^{31}$ La hipercapnia tisular aumenta cuando hay falla circulatoria secundaria a disfunción miocárdica, hipovolemia o sepsis.

El CO2 tiene un flujo arterial y un flujo venoso. La diferencia arteriovenosa de $\mathrm{pCO} 2$, ya sea de sangre venosa o de sangre venosa central ha sido considerada un marcador de la capacidad del sistema cardiovascular para eliminar el $\mathrm{CO} 2$ producido en los tejidos periféricos ${ }^{32}$ e incluso se ha demostrado que presenta correlación inversa con el índice cardíaco. ${ }^{33}$

Diversos estudios han demostrado que un delta $\mathrm{CO} 2>6 \mathrm{mmHg}$ persistente durante más de 12 horas podrían señalar la persistencia de hipoperfusión periférica, aun con valores normales de $\mathrm{SvcO} 2$, lo cual se asocia a un peor pronóstico y aumenta la mortalidad. ${ }^{34}$

Otros indicadores de perfusión tisular

En la actualidad se han desarrollado otros métodos que permiten evaluar la perfusión tisular en pacientes gravemente enfermos. La orthogonal polarization spectral (OPS) y sidestream darkfield imaging (SDF) son métodos de videomicroscopía que permiten obtener de imágenes a tiempo real de la microcirculación. ${ }^{35}$ También existen otras tecnologías como la capnografía sublingual ${ }^{36}$ y la espectroscopia de luz en el espectro cercano al infrarrojo (NIRS) ${ }^{37}$, sin embargo la mayoría de estos estudios no están desarrollados para su uso clínico, y no están disponibles en todas las salas de terapia intensiva, quedando restringidos a estudios experimentales.

Debido a que las características de la mucosa intestinal la hacen vulnerable a la disoxia, la tonometría gástrica se ha utilizado para evaluar la concentración de $\mathrm{CO} 2$ en el tejido y por tanto orientarnos en cuanto a la perfusión tisular. Algunos estudios multicéntricos demostraron que valores de delta $\mathrm{CO} 2$ por encima de $20 \mathrm{~mm} \mathrm{Hg}$ fueron capaces de discriminar a supervivientes de no-supervivientes en una población general de pacientes críticos, sin embargo estas mediciones no pueden aplicarse en todas las salas de cuidados intensivos debido a las dificultades técnicas que presenta su colocación y la interferencia de factores como la nutrición enteral. ${ }^{38}$

En cuanto a la a presión venosa central, no hay datos que respalden que su uso pueda indicarnos el estado de perfusión tisular y por tanto la práctica generalizada de utilizarla para guiar la terapia de líquidos no está justificada. ${ }^{39}$ 


\section{DISCUSIÓN}

La monitorización del estado de perfusión tisular per se no mejora el pronóstico del paciente crítico. Sin embargo, su utilización, ya sea como objetivo de reanimación o como herramienta en la toma de decisiones sí ha demostrado tener un impacto beneficioso sobre el pronóstico en la morbimortalidad del paciente.

La mayoría de los estudios consultados en esta revisión llevan a cabo la monitorización hemodinámica a través de una sola medición o como una media de un par de valores. En los pacientes con sepsis, este enfoque puede ser engañoso, ya que la evolución natural de la enfermedad y el tratamiento suelen producir cambios rápidos en la hemodinámica. Por lo tanto, es importante conocer las limitaciones de dicha monitorización a partir de la comprensión de las bases fisiopatológicas de las variables obtenidas.

$\mathrm{Al}$ elegir el método a utilizar para la monitoriza- ción de nuestro paciente, debemos tener en cuenta la tecnología disponible en nuestro servicio, la experiencia del personal de sanitario y costo-beneficio de su empleo.

\section{Conclusiones}

En el manejo del paciente críticamente enfermo que presenta shock, es importante revertir de manera precoz la hipoperfusión tisular para preservar la función de los tejidos y evitar el desarrollo posterior de la falla multiorgánica, la cual es una causa de mortalidad en estos pacientes.

En el momento actual, las variables más valiosas en cuanto a detección de hipoxia tisular son el lactato en sangre, la diferencia arteriovenosa de $\mathrm{CO} 2$ y las saturaciones venosas de oxígeno ( $\mathrm{SvO} 2$ y SvcO2), por tanto, la reanimación hemodinámica deberá estar dirigida a preservar la perfusión de los tejidos a través de la valoración integral de estos parámetros y su restitución a los valores fisiológicos.

\section{REFERENCIAS}

1.Rivers E, Nguyen B, Havstad S, Ressler J, Muzzin A, Knoblich $\mathrm{B}$, Peterson E, Tomlanovich M. Early goal directed therapy in the treatment of severe sepsis and septic shock. $N$ Engl J Med 2001;345:1368-1377.

2.Marik PE, Bellomo R. Stress hyperglycemia: an essential survival response! Critical Care. 2013;17(2):305.

3.F. Baigorri-González, JA. Lorente Balanza. Oxigenación tisular y sepsis. Med Intensiva 2005;29:178-84.

4.Varpula M, Tallgren M, Saukkonen K, et al. Hemodynamic variables related to outcome in septic shock. Intensive Care Med 2005;31:1066-1071.

5.Nava R. Zamudio P. Quiroz Y, Martínez I, Espinosa A, García A, Dominguez E. La disfunción mitocondrial como posible causa de la falla orgánica múltiple asociada a la sepsis severa. Rev Inst Nal Enf Resp Mex. 2009;22(1):37-47

6.A. Ochagavíaa, F. Baigorria, J. Mesquidaa, J.M. Ayuelab , A. Ferrándizc, X. García, M.I. Monged , L. Mateuc, C. Sabatiera, F. Clau-Terrée, R. Vichof, L. Zapatag, J. Maynarh, A. Gild. Monitorización hemodinámica en el paciente crítico. Recomendaciones del Grupo de Trabajo de Cuidados Intensivos Cardiológicos y RCP de la Sociedad Española de Medicina Intensiva, Crítica y Unidades Coronarias. Med Intensiva. 2014;38(3):154-169

7.Mesquida J, Borrat X., Lorente J.A., Masip J., Baigorri F. Objetivos de la reanimación hemodinámica. Med. Intensiva. 2011, 35(8): 499-508

8.Lavadenz LA, Pérez CM, et al. Diferencia veno-arterial de dióxido de carbono como predictor de mortalidad en pacientes en estado de shock, en Terapia Intensiva del Hospital Viedma, junio 2013-enero 2014. Rev Cient Cienc Méd. 2014. 17(2): 14-18.

9.Ocelotl PR, Valle RJ. Delta de CO2 como factor de riesgo de muerte en choque séptico. Rev. Asoc. Mex. Med. Crít. Ter. Intensiva. 2016 Abr. 30(1): 30-42.

10.Huang YCT. Monitoring oxygen delivery in the critically ill. Chest. 2005; 128: 554S-60S

11.Carrillo-Esper R. et al. Saturación venosa central, concep- tos actuales. Rev. Mex. De Anest. 2007.30 (3): 165-171.

12.Shapiro N. Howell M. Talmor D. et al. Serum lactate as predictor of mortality in emergency departament patients with infection. Ann Emerg Med. 2005; 45(5): 524-528

13.LeDoux D, Astiz M, Carpati C, Rackow E. Effects of perfusion pressure on tissue perfusion in septic shock. Crit Care Med. 2000;28:2729-32

14.Bourgoin A, Leone M, et al. Increasing mean arterial pressure in patients with septic shock: Effects on oxygen variables and renal function. Crit Care Med 2005; 33(4): 780-785.

15.Dellinger RP, Levy MM, Rhodes A, et al. Surviving Sepsis Campaign: international guidelines for management of severe sepsis and septic shock: 2012. Crit Care Med 2013;41:580637

16.Asfar P, Meziani F, Hamel JF, Grelon F, et al. SEPSISPAM Investigators High versus low blood-pressure target in patients with septic shock. N Engl J Med. 2014;370:1583-93.

17.Pope JV, Jones AE, Gaieski DF, Arnold RC, Trzeciak S, Shapiro NI, EMShockNet: Multicenter study of central venous oxygen saturation (ScvO2) as a predictor of mortality in patients with sepsis. Ann Emerg Med 2009, 55: 40-46.

18.Gutierrez G, Comignanni P, Huespe L, Hurtado FJ, Dubin A, Jha V, Arzani Y, Lazzeri S, Sosa L, Riva J, Kohn W, Suarez D, Lacuesta G, Olmos D, Mizdraji C, Ojeda A: Central venous to mixed venous blood oxygen and lactate gradients are associated with outcome in critically ill patients. Intensive Care Med 2008, 34: 1662-1668

19.Van Beest P, Wietasch G, Scheeren T, Spronk P, Kuiper M. Clinical review: use of venous oxygen saturations as a goal - a yet unfinished puzzle. Critical Care. 2011;15(5):232.

20.Varpula M, Karlsson S, Ruokonen E, Pettilä V: Mixed venous oxygen saturation cannot be estimated by central venous oxygen saturation in septic shock. Intensive Care Med 2006, 32: 1336-1343..

21.Chawla LS, Zia H, Gutierrez G, Katz NM, Seneff MG, Shah M: Lack of equivalance between central and mixed venous oxygen saturation. Chest 2004, 126: 1891-1896 
22.Reinhart K, Kuhn HJ, Hartog C, Bredle DL: Continuous central venous and pulmonary artery oxygen saturation monitoring in the critically ill. Intensive Care Med 2004, 30: 1572-1578.

23.Kopterides P, Bonovas S, Mavrou I, Kostadima E, Zakynthinos E, Armaganidis A: Venous oxygen saturation and lactate gradient from superior vena cava to pulmonary artery in patients with septic shock. Shock 2009, 31: 561-567.

24.Bakker J, Nijsten MW, Jansen TC. Clinical use of lactate monitoring in critically ill patients. Annals of Intensive Care. 2013;3:12.

25.Jansen TC, van Bommel J, Mulder PG, Rommes JH, Schieveld SJ, Bakker J. The prognostic value of blood lactate levels relative to that of vital signs in the pre-hospital setting: a pilot study. Crit Care.2008;3(6)

26.Dueñas C, Ortiz G, Mendoza R, Montes L. El papel del lactato en cuidado intensivo. Rev Chil de Med Intens 2016; 31(1): 13-22

27.Arnold RC, Shapiro NI, Jones AE, Schorr C, Pope J, Casner E, Parrillo JE, Dellinger RP, Trzeciak S, EMShockNet: Multicenter study of early lactate clearance as a determinant of survival in patients with presumed sepsis. Shock 2009, 32: 35-39.

28.Lee SW, Hong YS, Park DW, Choi SH, Moon SW, Park JS, et al. Lactic acidosis not hyperlactatemia as a predictor of in hospital mortality in septic emergency patients. Emerg Med J. 2008 Oct. 25 (10):659-65.

29.Mustafa I, Roth H, Hanafiah A, Hakim T, Anwar M, Siregar E, Leverve XM. Effect of cardiopulmonary bypass on lactate metabolism. Intensive Care Med. 2003;3(8):1279-1285

30.Vasquez G, García A, Montoya F. Utilidad del lactato sérico elevado como factor pronóstico de muerte en sepsis severa. Horiz Med 2015 (2): 35-40

31.Monnet, X., Julien, F., Ait-Hamou, N., Lequoy, M., Gosset,
C., Jozwiak, M., et al. Lactate and venoarterial carbon dioxide difference/arterial-venous oxygen difference ratio, but not central venous oxygen saturation, predict increase in oxygen consumption in fluid responders. Critical care medicine 2013; 41 (6): 1412-20.

32.Etulain GJ. Delta de dióxido de carbono para valorar perfusión tisular como predictor de mortalidad en choque séptico. Rev Mex Med Crit. 2011; 25(2): 66-70.

33.Bakker J, Vincent JL, Gris P, Leon M, Coffernils M, Kahn RJ. Veno arterial carbon dioxide gradient in human septic shock. Chest 2008; 101:509-515

34.Futier, E. Robin, E. Jabaudon, M. Guerin, R. Petit, A. Bazin $\mathrm{JE}$, Vallet, $\mathrm{B}$ Central venous $\mathrm{O} 2$ saturation and venous to arterial $\mathrm{CO} 2$ difference as complementary tools for goal directed therapy during high risk surgery. Crit Care. 2010; 14(5):193.

35.Myers DE, Anderson LD, Seifert RP, et al. Noninvasive method for measuring local hemoglobin oxygen saturation in tissue using wide gap second derivative nearinfrared spectroscopy. J Biomed Opt. 2005; 10: 034017.

36.Marik PE, Bankov A. Sublingual capnometry versus traditional markers of tissue oxygenation in critically ill patients. Crit Care Med. 2003; 31: 818-22.

37.Crookes BA, Cohn SM, Bloch S, Amortegui J, Manning R, Li $P$, et al. Can near-infrared spectroscopy identify the severity of shock in trauma patients? J Trauma. 2005; 58: 806-16.

38.Levy B, Gawalkiewicz P, Vallet B, et al. Gastric capnometry with air-automated tonometry predicts outcome in critically ill patients. Crit Care Med. 2003; 31: 474-80.

39.Marik PE, Cavallazzi R. Does the central venous pressure predict fluid responsiveness? An updated meta-analysis and a plea for some common sense. Crit Care Med. 2013 Jul;41(7):1774-81 